\title{
1 Facile mechanical-induced functionalization of 2 hexagonal boron nitride and its application as 3 vehicles for antibacterial essential oil
}

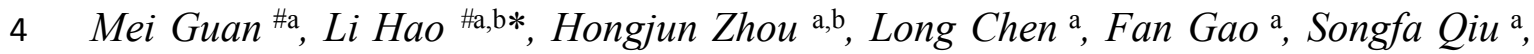

5 Hongjun Zhou ${ }^{\mathrm{a}, \mathrm{b} *}$, Huayao Chen ${ }^{\mathrm{a}, \mathrm{b}}$, Xinhua Zhou ${ }^{\mathrm{a}, \mathrm{b} *}$

6 aSchool of Chemistry and Chemical Engineering, Zhongkai University of Agriculture 7 and Engineering, Guangzhou, Guangdong, 510225, P. R. China;

8 bKey Laboratory of Agricultural Green Fine Chemicals of Guangdong Higher

9 Education Institution, Zhongkai University of Agriculture and Engineering, Guangzhou,

10 Guangdong, 510225, P. R. China

\#Mei Guan and Li Hao contributed equally to this work.

Corresponding authors:

* Li Hao, Phone: +86-20-89003114, E-mail: haoli20@tju.edu.cn

* Hongjun Zhou, Phone: +86-20-89003114, E-mail: hongjunzhou@163.com

* Xinhua Zhou, Phone: +86-20-89003114, E-mail: cexinhuazhou@163.com 


\section{S2 Experimental Section}

\section{S2.1 Materials}

Hexagonal boron nitride (h-BN) powder (99.9\%, with average size of 1-2 $\mu \mathrm{m})$ was purchased from Aladdin Chem. Co. Ltd. (Shanghai, China). Soy protein isolate (SPI, $\geq$ 95\%, aqueous soluble) was provided by Tianli Food Co. Ltd. (Anyang, China) and obtained by extraction of de-fatted soy protein powder. Sodium carboxymethyl cellulose (CMC, USP grade) with a viscosity of $300-800 \mathrm{mPa} \cdot \mathrm{s}$ was procured from Aladdin Chem. Co. Ltd. (Shanghai, China). Ethanol (anhydrous), sodium hydroxide $(\mathrm{NaOH}, \geq 97.0 \%)$, and hydrochloric acid $(\mathrm{HCl}, 37 \%)$ were purchased from Tianjin Damao Chemical Reagent Co., Ltd. (Tianjin, China). Tea tree oil (TTO) was obtained from Aladdin Chem. Co. Ltd. (Shanghai, China) and used directly without further purification. Deionized water was used in all experiments.

\section{S2.2 Simultaneously exfoliation and functionalization of hexagonal boron nitride}

The mechanochemical concept is inspired by a literature and improved with our own ideas. ${ }^{1}$ In a typical polysaccharide-protein-assisted mechanochemical process, polysaccharide $(\mathrm{CMC})$, protein $(\mathrm{SPI})$, and bare h-BN were added at a mass ratio of 1:1:4 and loaded into a tank with agate balls in two different diameters of $10 \mathrm{~mm}$ (100 $\mathrm{g})$ and $1 \mathrm{~mm}(10 \mathrm{~g})$, and then the tank was rotated under $10 \mathrm{~Hz}(600 \mathrm{r} / \mathrm{min})$ for $12 \mathrm{~h}$ in a planetary ball milling (QM-3SP04, Nanjing Chi Shun Technology Development Co., Ltd, Jiangsu, China) to induce polysaccharide-protein-assisted exfoliation and functionalization of boron nitride nanosheets (BNNS) with SPI and CMC. Afterwards, 
the milled products were washed with deionized water several times to remove nongrafted moieties thoroughly and then centrifuged at $4000 \mathrm{rpm}$ for a certain time to obtain the entire precipitates. Next, the precipitates consisting of BNNS grafted with SPI and CMC were further dried at $40{ }^{\circ} \mathrm{C}$ for $24 \mathrm{~h}$. The resultant exfoliated and functionalized BNNS was acquired and denoted as CMC-SPI-BNNS composites. In comparison, solely CMC and SPI was blended with bare h-BN powder at a mass ratio of $2: 1$, then ball milled at a power of $10 \mathrm{~Hz}(600 \mathrm{r} / \mathrm{min})$ for $12 \mathrm{~h}$ in the same planetary ball milling, the obtained products were defined as CMC-BNNS and SPI-BNNS. As a control, bare h-BN without any additives was milled under the same conditions as aforementioned, and denoted as BNNS.

The bare h-BN and functionalized BNNS powders (i.e. BNNS, CMC-BNNS, SPI-BNNS, CMC-SPI-BNNS) were directly dispersed in water, ethanol, and ethanolwater mixture $(55 / 45 \mathrm{w} / \mathrm{w})$ solutions at a concentration of $1 \mathrm{mg} \cdot \mathrm{mL}^{-1}$ under bath sonication (JP-060, Skymen Cleaning Instrument Co. Ltd, Shenzhen, China) at a power of $360 \mathrm{~W}$ to observed the dispersibility, stability, and Tyndall effect pathway generated by irradiation with a laser point by standing for 31 days at room temperature.

\section{S2.3 Functionalized boron nitride-based materials as vehicles for TTO}

The loading of TTO into boron nitride-based materials as vehicles, including hBN, BNNS, CMC-BNNS, SPI-BNNS, and CMC-SPI-BNNS was obtained via an incubation method, slightly modified according to our previous work..$^{2}$ In a typical procedure, $50 \mathrm{mg}$ of boron nitride-based materials was placed into funnels with filter 
papers, respectively, and then $5 \mathrm{~mL}$ of tea tree oil was immersed into the funnels with boron nitride-based materials, which was placed to stand for $24 \mathrm{~h}$ in a dark and mild space, avoiding the oxidation of TTO. Afterwards, the free TTO and TTO on the surface was removed entirely by filtering out and evaporating after $24 \mathrm{~h}$, hence, the TTO-loaded boron nitride-based materials were acquired and designated as TTO@h-BN, TTO@BNNS,TTO@CMC-BNNS, TTO@SPI-BNNS, TTO@CMC-SPI-BNNS.The amount of TTO loaded into boron nitride-based materials was quantified using thermogravimetric analyzer (Mettler Toledo, Switzerland) and calculated according to the weight loss, wherein weight loss peak of TTO usually occurred at $100-110^{\circ} \mathrm{C}$.

\section{S2.4 Characterization}

Fourier Transform Infrared (FTIR) spectroscopy was performed to characterize pristine h-BN, BNNS, CMC-BNNS, SPI-BNNS, and CMC-SPI-BNNS in the range of 4000-400 $\mathrm{cm}^{-1}$ using $\mathrm{KBr}$ pellet method through a Spectrum 100 Fourier Infrared spectrometer (Perkin Elmer Inc., USA).

Raman spectroscopy (DXR2, Thermo Fisher, USA) measurements were conducted to study the interactions within CMC, SPI, and BNNS. During the test, the detector was $785 \mathrm{~nm}$ and the slit width was $20 \mu \mathrm{m}$.

Ultraviolet-visible diffuse reflectance spectroscopy (UV-Vis DRS) measurements were carried out using a UV-3600 spectrophotometer (Shimadzu, Japan) equipped with an integrating sphere attachment $\mathrm{BaSO}_{4}$ as a reflectance standard within 200-2000 nm. 
X-ray photoelectron spectroscopy (XPS) experiments were carried out with a KAlpha spectrometer (Thermo Scientific, USA) under vacuum using monochromatic Al $\mathrm{K} \alpha \mathrm{X}$-rays to obtain the surface elements.

X-ray diffraction (XRD) patterns were recorded on a D8 diffractometer (Bruker AXS $\mathrm{GmbH}$, Germany) with a $\mathrm{Cu} \mathrm{K} \alpha$ radiation and a graphite monochromator in angular range of $2 \theta$ between 5 and $90^{\circ}$.

The morphology, shape and size were observed by scanning electron microscope (SEM, ZEISS, German). In the scanning electron microscope test, a small amount of sample powder was dipped in a capillary tube and evenly coated on the sample table. Before testing, it was necessary to spray gold for $45 \mathrm{~s}$ to increase the conductivity of the sample, and then the test was performed to obtain the morphological characteristics. Besides, transmission electron microscope (TEM, TF20 2100F, JEOL, Japan) and high resolution TEM (HRTEM) images were also used to characterize the morphology, shape, and size.

$\mathrm{N}_{2}$ adsorption/desorption isotherms were obtained using ASAP2460 adsorption instrument (Micromeritics, USA) at $77 \mathrm{~K}$. Before $\mathrm{N}_{2}$ adsorption, the samples were degassed at $353 \mathrm{~K}$ for $12 \mathrm{~h}$ under vacuum.

Zeta potential measurements were performed by dispersing and diluting samples in water and carried out via a 90 Plus PALS particle size and zeta potential analyzer (Bruker Corporation, USA) to compare the colloidal stability. 

analyzer (Mettler Toledo, Switzerland) under $\mathrm{N}_{2}$ atmosphere with a flow rate of 20 $\mathrm{mL} \cdot \mathrm{min}^{-1}$, heating from $40{ }^{\circ} \mathrm{C}$ to $600{ }^{\circ} \mathrm{C}$ at a heating rate of $10{ }^{\circ} \mathrm{C} \cdot \mathrm{min}^{-1}$.

Differential scanning calorimetry (DSC) was carried out using a Q20 differential scanning calorimeter (TA Instruments, USA) by heating from $40{ }^{\circ} \mathrm{C}$ to $160{ }^{\circ} \mathrm{C}$ at a heating rate of $10^{\circ} \mathrm{C} / \mathrm{min}$ under a nitrogen atmosphere $(50 \mathrm{~mL} / \mathrm{min})$.

\section{S2.5 Study of sustained release performance and release kinetics of TTO from} boron nitride-based materials

The sustained release behavior was obtained with a thermogravimetric analyzer (Mettler Toledo, Switzerland) at a constant temperature of $40{ }^{\circ} \mathrm{C}$ releasing for $10 \mathrm{~h}$ to compare the cumulative release trend for various TTO-loaded samples and further study their release kinetics using different models. According to the data obtained from TGA curves, the cumulative release percentage was calculated using formula S1, where $M_{0}$ (mg) is the initial weight, $M_{\mathrm{t}}(\mathrm{mg})$ represents the remaining weight at intervals after TTO releasing. $L C(\%)$ is loading capacity, indicating the amount of TTO carried by boron nitride-based materials, which also calculated from TGA weight loss curves as aforementioned. 
127

determined by agar plate diffusion method. ${ }^{3}$ Escherichia coli $($ E. coli $)(0.1 \mathrm{~mL})$ was inoculated into Luria Bertani (LB) broth with an initial cell density of $1 \times 10^{5} \mathrm{CFU} \cdot \mathrm{mL}^{-1}$ and incubated at $37{ }^{\circ} \mathrm{C}$ for $24 \mathrm{~h}$. Then, E. coli was evenly spread on agar plates. Afterwards, $30 \mathrm{mg}$ of TTO-loaded boron nitride-based powders was placed into a $6 \mathrm{~mm}$ diameter hole in the center of the ager plates through an Oxford cup and then all Petri dishes for testing were incubated at $37^{\circ} \mathrm{C}$ for $24 \mathrm{~h}$. As a control, equivalent pure TTO calculated in terms of loading capacity of TTO-loaded boron nitride-based powders (approximately $9.7 \mathrm{mg}$ ) was also used to investigate the antibacterial activity. The inhibition of bacteria with TTO-loaded boron nitride-based materials was evaluated by measuring the diameter of bacteriostatic ring, from which the samples exhibited better antibacterial activity if the inhibitory region larger. ${ }^{4}$

\section{S2.7 Study on the minimal inhibitory concentration of boron nitride-based carriers loaded with TTO against $E$. coli}

Antibacterial activity of TTO-loaded boron nitride materials was quantitatively analyzed by determining the minimal inhibitory concentration (MIC) followed a previous reported method.$^{5}$ In a typical process, a certain amount of TTO-loaded boron nitride-based powders was dispersed into $20 \mathrm{~mL}$ of sterilized nutrient agar under ultrasonication at $45^{\circ} \mathrm{C}$ for 30 min to obtain dispersions with various concentrations including $0.1 \mathrm{mg} \cdot \mathrm{mL}^{-1}, 0.2 \mathrm{mg} \cdot \mathrm{mL}^{-1}, 0.4 \mathrm{mg} \cdot \mathrm{mL}^{-1}, 0.6 \mathrm{mg} \cdot \mathrm{mL}^{-1}, 0.8 \mathrm{mg} \cdot \mathrm{mL}^{-1}$. Then, the dispersions were poured into $0.1 \mathrm{~mL}$ of $E$. coli suspension at a concentration of $1 \times 10^{5}$ 
147 148 for $24 \mathrm{~h}$ in the dark. And then the numbers of colonies on culture dishes was observed 149 and the corresponding MIC value was determined by measuring the minimal 150

$\mathrm{CFU} \cdot \mathrm{mL}^{-1}$ and mixed uniformly in shaking. Next, the mixture was incubated at $37^{\circ} \mathrm{C}$ concentration of sample solution upon the cells with no bacterial growth of E. coli. ${ }^{5,6}$ The antibacterial rate (\%) is calculated from the inhibition number of $E$. coli cell colonies by TTO samples comparing to blank without any TTO samples, which can be calculated according to formula (S2), where $C_{\text {blank }}$ represents the number of bacterial cell colonies without any TTO samples after incubation for $24 \mathrm{~h}$, and $C_{\mathrm{A}}$ represents the number of bacterial cell colonies after 24 h of incubation with pure TTO, TTO@h-BN, TTO@BNNS, TTO@SPI-BNNS, TTO@CMC-BNNS, TTO@CMC-SPI-BNNS, respectively.

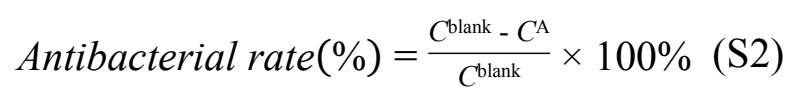

\section{S2.8 Statistical analysis}

All the antibacterial tests in this study were conducted in triplicates. The inhibition rate of TTO against $E$. coli as a control, evaluated the difference in inhibition of $E$. coli between the samples by difference factor $\left(f_{1}\right)$ and similarity factor $\left(f_{2}\right)$, and $f_{1}$ and $f_{2}$ were obtained from fomula (S3) and (S4). ${ }^{7,8}$

$$
f_{1}=100\left[\frac{\left(\sum_{t=1}^{n}\left|R_{i}-T_{i}\right|\right)}{\sum_{t=1}^{n} R_{i}}\right](\mathrm{S} 3)
$$




$$
f_{2}=50 \log \left\{\left[1+\left(\frac{1}{n}\right) \sum_{t=1}^{n}\left(R_{i}-T_{i}\right)^{2}\right]^{-0.5} \times 100\right\}(\mathrm{S} 4)
$$
sample at specific time intervals.

\section{S3 Results and discussion}
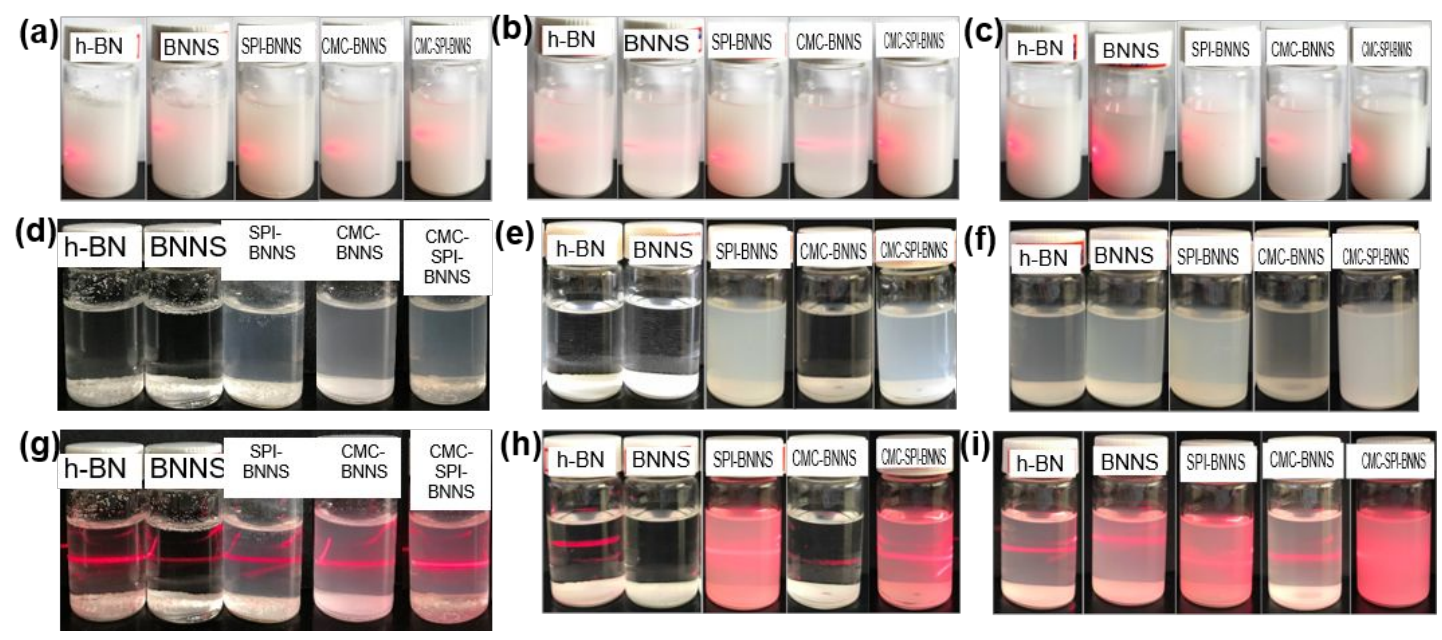

Figure S1. Tyndall effect images of pristine h-BN, BNNS, SPI-BNNS, CMC-BNNS, and CMC-SPI-BNNS fresh suspension in water (a), ethanol (b), and ethanol-water (c); photos of above samples suspensions in water (d), ethanol (e), and ethanol-water (f) after standing for 31 days demonstrating stability; and corresponding Tyndall effect images of samples dispersed in water (g), ethanol (h), and ethanol-water (i) after standing for 31 days. 

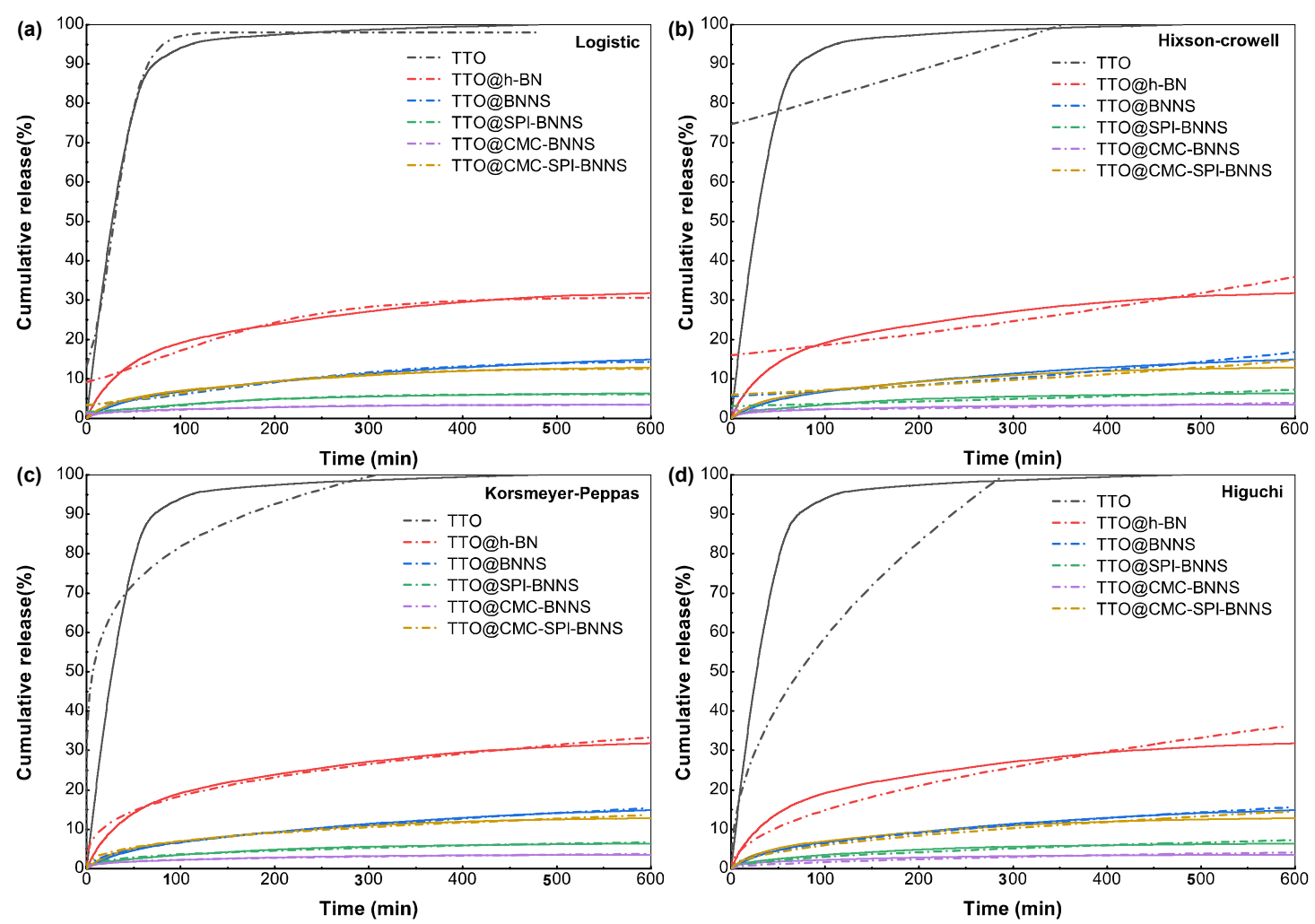

Figure S2. Model fitting curves for pure TTO release, and TTO release from vehicles, including h-BN, BNNS, SPI-BNNS, CMC-BNNS, CMC-SPI-BNNS, in which the solid lines represent the original experimental data and the dash-dot lines represent the

181 fitting curve. 


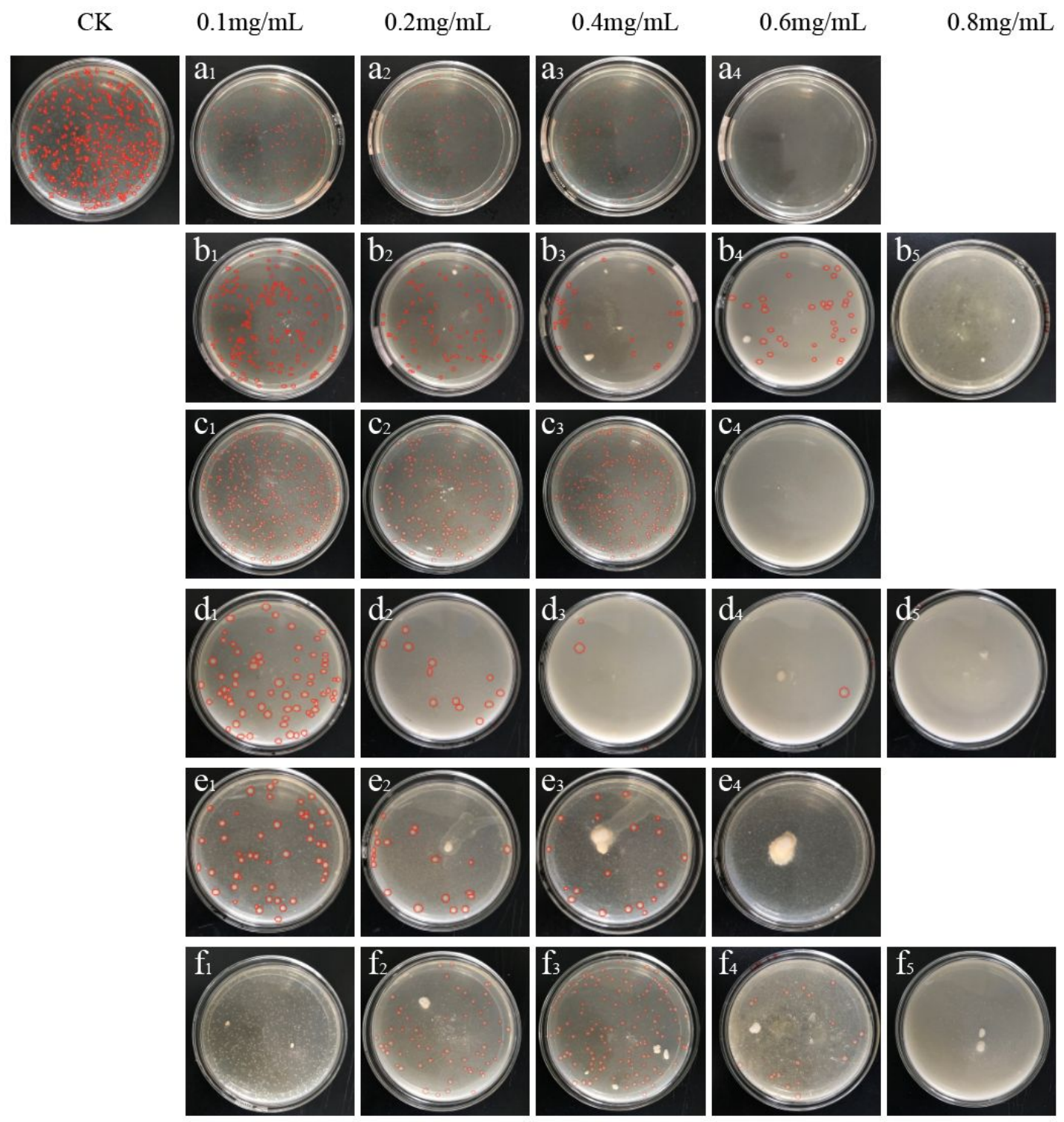

Figure S3. Colony count results of the bacterial suspension containing TTO loaded BN-based materials under the minimal inhibitory concentration against $E$. coli growth: CK represents control blank without any TTO samples, and $\left(a_{1}-a_{4}\right)$ pure TTO, $\left(b_{1}-b_{5}\right)$ TTO@h-BN, ( $\left.\mathrm{c}_{1}-\mathrm{c}_{4}\right)$ TTO@BNNS, $\left(\mathrm{d}_{1}-\mathrm{d}_{5}\right)$ TTO@SPI-BNNS, (e $\left.\mathrm{e}_{1}-\mathrm{e}_{4}\right)$ TTO@CMCBNNS, $\left(\mathrm{f}_{1}-\mathrm{f}_{5}\right)$ TTO@CMC-SPI-BNNS, with different concentrations, respectively.

The antibacterial activity assay experiments for CMC, SPI, CMC-SPI and BNNS were also provided and their inhibition zones against $E$. coli growth is presented in Figure S4. And it can be clearly observed that neither the carrier nor the synthetic monomer shows an inhibitory region against E. coli. Among them, the Figure S4 d, f and g partially swelled due to the existence of CMC, but the results of the punching test 
that never swelled could prove that the carrier did not have antibacterial properties against $E$. coli. Based on this, it can be determined that the antibacterial effect of BNbased materials loaded with TTO on E. coli is produced by TTO.
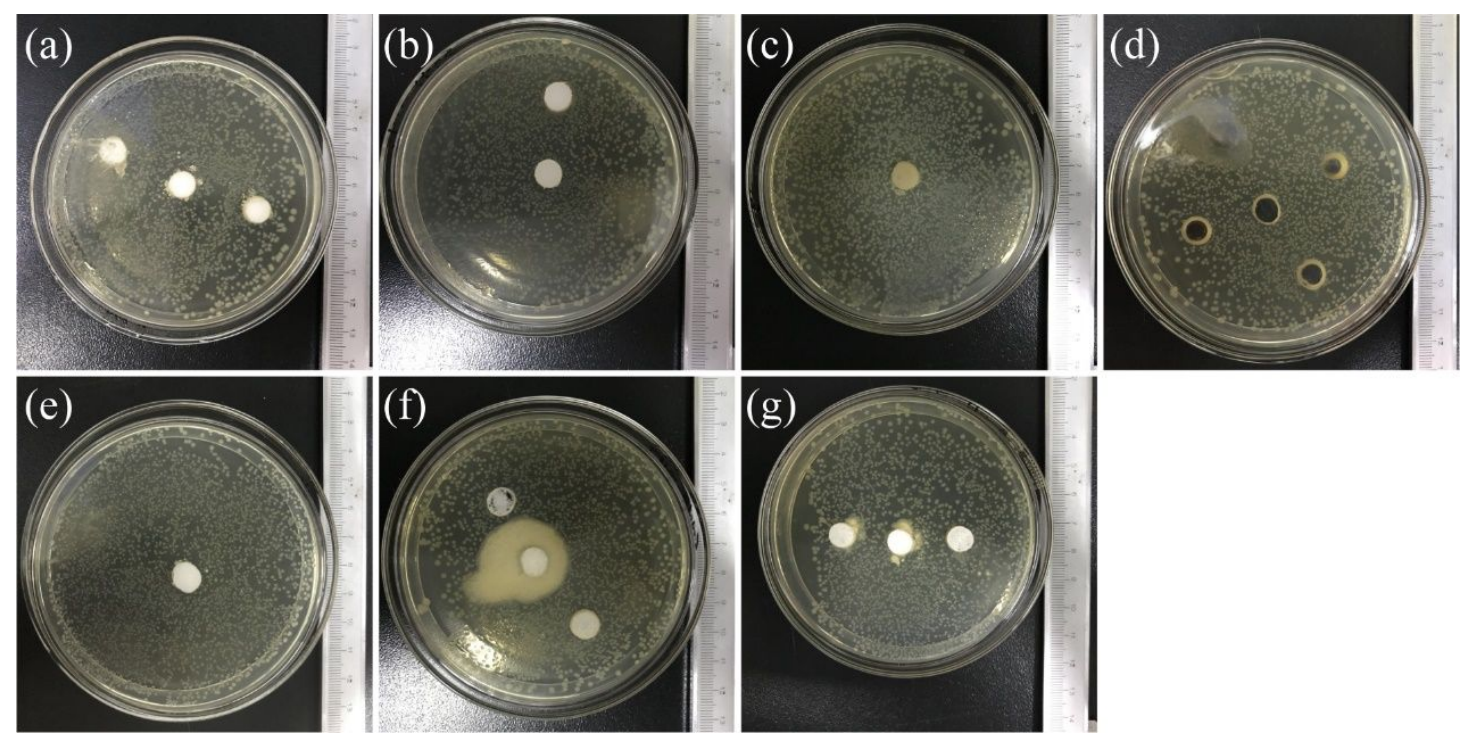

Figure S4. Inhibition zones against $E$. coli growth by BN-based materials after cultured for 24 h:(a) h-BN, (b) BNNS, (c) SPI, (d) CMC, (e) SPI-BNNS, (f) CMC-BNNS, (g) CMC-SPI-BNNS.

The differences and similarities in the suppression zone were statistically analyzed to inhibition of $E$. coli by tea tree essential oil in different samples. The results of $f_{1}$ and $f_{2}$ are shown in Table S1. When $f_{1}>15$ or $f_{2}<50$, the tea tree essential oils between reference and test samples had significant differences in the inhibition rate of E. coli. h-BN and CMC-BNNS inhibition rate of $E$. coli after loading TTO, whose $f_{1}$ were $<$ $15, f_{2}$ were $>50$, and indicated no significant differences between the test and the reference. For BNNS, SPI-BNNS and CMC-SPI-BNNS with loading TTO, the calculated $f_{1}$ were $>15$ and $f_{2}$ were $<50$, indicating that these samples are significantly different from the reference. 
Table S1. Results of the statistical analysis.

\begin{tabular}{cccc}
\hline Reference sample & Control sample & $f_{1}$ & $f_{2}$ \\
\hline \multirow{2}{*}{ TTO } & TTO@h-BN & 10.02 & 52.35 \\
& TTO@BNNS & 35.96 & 31.01 \\
& TTO@SPI-BNNS & 34.91 & 20.57 \\
& TTO@CMC-BNNS & 3.158 & 71.59 \\
& TTO@CMC-SPI-BNNS & 49.59 & 17.05 \\
\hline
\end{tabular}

\section{REFERENCES}

212

1. Chen, S.; Xu, R.; Liu, J.; Zou, X.; Qiu, L.; Kang, F.; Liu, B.; Cheng, H. M., Simultaneous production and functionalization of boron nitride nanosheets by sugar-assisted mechanochemical exfoliation. Adv. Mater. 2019, 31 (10), e1804810, DOI 10.1002/adma.201804810

2. Gao, F.; Zhou, H.; Shen, Z.; Qiu, H.; Hao, L.; Chen, H.; Zhou, X., Synergistic antimicrobial activities of tea tree oil loaded on mesoporous silica encapsulated by polyethyleneimine. J. Disper. Sci. Technol. 2019, 1-13, DOI 10.1080/01932691.2019.1637755

3. Carson, C. F.; Riley, T. V., Antimicrobial activity of the major components of the essential oil of Melaleuca alternifolia. J. Appl. Biomater. 1995, 78 (3), 264-269, DOI 10.1111/j.1365-2672.1995.tb05025.x

4. Ge, M.; Li, J.; Song, S.; Meng, N.; Zhou, N. J., Development and antibacterial performance of silver nanoparticles-lecithin modified montmorillonite nanoparticle hybrid. Appl. Clay Sci. 2019, 183, 105334, DOI 10.1016/j.clay.2019.105334

5. Yu, Q.; Xu, X.; Wang, C.; Ma, Y.; Hui, D.; Zhou, Z., Remarkably improvement in antibacterial activity by synergistic effect in $\mathrm{n}-\mathrm{Cu} @ \mathrm{~T}-\mathrm{ZnO}$ nanocomposites. Compos. Part B: Eng. 2017, 110, 32-38, DOI 10.1016/j.compositesb.2016.10.085

6. Ma, P.; Jiang, L.; Yu, M.; Dong, W.; Chen, M.; Green antibacterial nanocomposites from poly(lactide)/poly(butylene adipate-coterephthalate)/nanocrystal cellulose-silver nanohybrids. ACS Sustainable Chem. Eng. 2016, 4 (12), 6417-6426, DOI 10.1021/acssuschemeng.6b01106

7. Chen, L.; Zhou, X.; Lin, G.; Chen, H.; Hao, L.; Zhou, H., Synthesis of $\mathrm{pH}$-responsive isolated soy protein/carboxymethyl chitosan microspheres for sustained pesticide release. J. Appl. Polym. Sci. 2019, 137 (6), 48358, DOI 10.1002/app.48358 8. Lin, G.; Chen, X.; Zhou, H.; Zhou, X.; Xu, H.; Chen, H., Elaboration of a feather keratin/carboxymethyl cellulose complex exhibiting $\mathrm{pH}$ sensitivity for sustained pesticide release. J. Appl. Polym. Sci. 2019, 136 (10), 47160, DOI 10.1002/app.47160 